\title{
Risk Analysis of Aedes triseriatus in China
}

\author{
Jingyuan $\mathrm{Liu}^{1}$, Xiaoguang $\mathrm{Ma}^{1,{ }^{1}}$, Zhihong $\mathrm{Li}^{2}$, Xiaoying $\mathrm{Wu}^{1}$, and $\mathrm{Nan} \mathrm{Sun}^{3}$ \\ ${ }^{1}$ Institute of Health Quarantine,Chinese Academy of Inspection and Quarantine, \\ Beijing, P. R. China \\ ${ }^{2}$ College of agriculture and biotechnology, China Agricultural University, Beijing, P. R. China \\ ${ }^{3}$ Shanghai Entry-Exit Inspection and Quarantine Bureau, P. R. China \\ yyuan_108@sina.com
}

\begin{abstract}
Aedes triseriatus(Say), the eastern treehole mosquito (Diptera: Culicidae), is an important medical vector transmitting the LaCrosse encephalitis(LAC) and West Nile Virus, distributed in north America, which ever caused the LAC epidemic in east and central America. With the medical-vectors intercepted data of Sino-North America trade at port, it was confirmed that it is possible for A. triseriatus to invade China by cargoes, imported timbers, containers, etc. The life cycle of $A$. triseriatus is closely related to the temperature and humidity. Three models were used. CLIMEX model was used to analyze the possible colonized area of $A$. triseriatus in China, the epidemic risk of LAC encephalitis transmitted by $A$. triseriatus was analyzed by MaxEnt model, and ArcGIS9.2 was used to analyze the model results. The purpose of this research is to establish a superposition model on the risk analysis of medical-vectors. The result shows that the eastern treehole mosquito could colonize in China. The high risk areas of LAC encephalitis are in Yunnan, Sichuan, Guizhou, Chongqing, Hubei, Anhui, Jiangsu, Zhejiang, Jiangxi, Hunan, Guangxi, Guangdong, Fujian, Hainan, Taiwan; South of Shanxi, Henan, Shandong; Southeast of Liaoning provinces; The medium risk areas covers most of Shanxi, Shaanxi, Hebei, Tianjin, Jilin, Heilongjiang; South of Xizang, Gansu, Ningxia, Qinghai; North of Sichuan, Liaoning, Shandong, Henan, Middle of Neimeng and parts of southwest Xinjiang provinces. The low risk area includes north of Xizang, Ningxia; South of Qinghai; Middle of Gansu, Neimeng, North of Ningxia, parts of southwest Xinjiang provinces.
\end{abstract}

Keywords: Medical vector; Risk Analysis; CLIMEX model; MaxEnt model; ArcGIS software; Model superposition.

\section{Introduction}

The relevant laboratory study shows that conditions, A. triseriatus is a vector of several viruses such as yellow fever, eastern encephalitis, Venezuelan encephalitis and western encephalitis. It is also the vector of canine heartworm disease. A. triseriatus propagates in water, particularly accumulated water in treeholes and tires, and can also survive in artificial vessels where there is accumulated water, so the mosquito

Corresponding author. 
can survive in any place where accumulated water is available ${ }^{[2]}$. With increase in trade between China and North America, A. triseriatus is likely to invade China through cargoes, imported timbers and containers. It is a medical vector which may poses a serious threat to China. Therefore, it is imperative to predict the potential distribution scope of the mosquito in China with a view to preventing its invasion. $A$. triseriatus will colonization in an area is closely related to climatic factors such as temperature, humidity and rainfall, so the software CLIMEX based on the phenologic and climatic conditions is selected to predict its potential distribution in China. The software MaxEnt based on the maximum entropy algorithm is used to predict the possible spread areas of LAC encephalitis transmitted by $A$. triseriatus.

This paper provides s a risk analysis of A. triseriatus based on a superposition model.

\section{Materials and Methods}

\subsection{Materials}

\subsubsection{Analysis of Limiting Factors for Distribution of Aedes triseriatus}

The life cycle of A. triseriatus includes four stages: egg, larva, pupa, and adult. Adult A. triseriatus mosquitoes spawn on water surface and has one or two generations each year, depending on environmental conditions, especially rainfalls ${ }^{[2]}$. In Michigan of the US, the eggs hatch into larvae in March to April. There are four larval instars, and fourth instar larvae will moult into pupae. In northern states of the US, adult mosquitoes start appearing from mid-July to September. The eggs will enter a state of diapause under appropriate conditions, and in the southern US, this insect overwinters in mature diapause larvae. During diapause, larvae can move freely to seek foods, but non-diapause larvae move slowly and eat less. In Toronto, Canada, first instar larvae appear in mid-March, with water temperature at $4.1-9.7^{\circ} \mathrm{C}$, second instar larvae start appearing in mid-April, and larvae start pupating in mid-May. Adults appear in June or July. The second-generation larvae appear in mid-July to mid-August, the second-generation population over-winters in larval stage before there life cycle ends ${ }^{[1]}$. Adult male mosquitoes appear earlier than female mosquitoes, and female mosquitoes start seeking foods 3 days after appearing. This mosquito prefers to bite mammals and is sensitive to light. The relevant study indicates that the photoperiod is a major factor that induces its overwintering diapause and a low-temperature environment can strengthen photoperiod induction ${ }^{[3]}$.The state of diapauses will end under long-lasting sunlight ${ }^{[4]}$. The photoperiod for inducing diapuase varies in different latitudes ${ }^{[5]}$.

A. triseriatus has strong resistance to low temperature. In 1972, M Jail studied the influence of different gradients of temperature on its growth under laboratory conditions. The study pointed out that the temperature for the larvae of this mosquito to start growing normally is $9^{\circ} \mathrm{C}$. In water below $4^{\circ} \mathrm{C}$ or above $40^{\circ} \mathrm{C}$, all A. triseriatus will die within 3 days. Under $6^{\circ} \mathrm{C}, 8^{\circ} \mathrm{C}$ and $38^{\circ} \mathrm{C}$, the larvae can grow to second instars, but will not continue growing. When the water temperature reaches $40^{\circ} \mathrm{C}$, the larval mortality rate is still very high, with only $31 \%$ larvae survived. Obviously, high 
temperature limits its growth. In 2007, the study by Williams et al. in Canada stated that $A$. triseriatus might survive in southern Canada, and under natural conditions, it could spawn in water lower than $10^{\circ} \mathrm{C}$ and first instar larvae were found in water of $0.7^{\circ} \mathrm{C}$. Obviously, temperature is a main factor controlling the southern and northern boundary in distribution of $A$. triseriatus.

The eggs, larvae and pupae of $A$. triseriatus live in water and will not survive under overly dry environment. Strong rainfall will wash away A. triseriatus on water surface. Therefore, ambient humidity and rainfall limit its distribution to a certain extent.

\subsubsection{Model}

The version number of the CLIMEX model is 2.0, and that of the MaxEnt model is 2.3, which can be downloaded directly via network (source website: http://www.cs.princeton.edu/ schapire/max-ent/). The GIS analysis software is ESRI ArcGIS9.2.

\subsubsection{Data}

The CLIMEX model has 2414 global meteorological stations including 86 Chinese stations, and meteorological data can be purchased from the Earth System Scientific Data Sharing Network (http: //www.geodata.cn/Portal/) according to the research needs to add Chinese meteorological stations. The environmental data necessity for the MaxEnt model is world eco-environmental data provided by Desktop GARP platform, including data such as elevation, climate and type of vegetation etc. The maps ArcGIS analysis come from the National Fundamental Geographic Information System (http://nfgis.nsdi.gov. cn/).

\subsection{Methods}

The data input in the CLIMEX model are biological data of eastern treehole mosquito including climatic factors such as temperature, humidity, illumination and stress. The model calculates the ecoclimatic index EI which reflects the probability of colonization of a species in a region. We can predict the probability of colonization of a species in a new habitat by adjusting the parameters so that the distribution of EI values maximally matches the present distribution of $A$. triseriatus. The inverse distance weight interpolation method of ArcGIS can carry out interpolation analysis of EI values to demonstrate more intuitionistically the potential distribution of A. triseriatus in China.

The MaxEnt model can calculate the survival requirements of LAC virus using environmental data based on its known distribution and the eco-environmental data. Known distribution data was listed as the input data according the published references. $80 \%$ of the input data was used to build the requested model, and the left $20 \%$ was used to modify it. Run the model to achieve the potential spreading scope of LAC in a new habitat.

Space analysis of ArcGIS software was used to analyze the possible colonization of A. triseriatus if it is spread into China and the potential distribution scope of LAC virus transmitted by it in China, obtained the risk analysis result for A. triseriatus in China. 


\section{Result}

\subsection{Potential Distribution Result of $A$. triseriatus in China}

The preliminary parameters acquired according to biological data were adjusted match the existing distribution. The adjusted parameters are shown in the following table.

Table 1. CLIMEX parameters of Aedes triseriatus

\begin{tabular}{lr}
\hline Parameter description & Value \\
\hline Lower temperature threshold DV0 & 6 \\
Lower optimum temperature DV1 & 15 \\
Upper optimum temperature DV2 & 28 \\
Upper temperature threshold DV3 & 38 \\
Lower soil moisture threshold SM0 & 0.2 \\
Lower optimal soil moisture SM1 & 0.7 \\
Upper optimal soil moisture SM2 & 1.2 \\
Upper soil moisture threshold SM3 & 2 \\
Cold stress temperature threshold TTCS & 5 \\
Cold stress accumulates rate THCS & -0.00001 \\
Dry stress threshold SMDS & 0.1 \\
Dry stress rate HDS & -0.00001 \\
\hline
\end{tabular}

According to the adjusted parameters, the potential distribution of A. triseriatus in China was predicted. The interpolation result of ArcGIS was compared with the actual distribution in North America to carry out classification. The result shows that this Aedes mosquito is widely distributed in China, covering almost regions in our country. According to its distribution in North America, the EI values are divided into four risk levels: high, moderate, low and no risk. Among them, the EI value is above 30 and marked in red in the areas such as Yunnan, Guizhou, Chongqing, Guangxi, Guangdong, Hong Kong, Hainan, Hunan, Hubei, Jiangxi, Fujian, Zhejiang, Anhui, Jiangsu, Taiwan, east Liaoning, southeast Shandong, south Henan, south Shaanxi, southeast Sichuan and south Tibet. This indicates that there is a high risk of colonization of $A$. triseriatus in the above areas. The EI value is during 9-30 and marked in orange in the areas such as Heilongjiang, Jilin, Hebei, Shanxi, Liaoning, northeast Shandong, north Henan, north Shanxi, south Gansu, south Ningxia, northwest Sichuan, south Tibet, northeast Inner Mongolia, southeast Qinghai and northwest Xinjiang. This shows that there is a possibility of colonization of $A$. triseriatus in the above areas. The EI value is during 1-9 and marked in yellow in the areas such as North Tibet, central Qinghai, central Gansu, central Inner Mongolia, north Ningxia and northeast Xinjiang. This indicates that A. triseriatus can survive in the above areas and there is a risk of its colonization. 


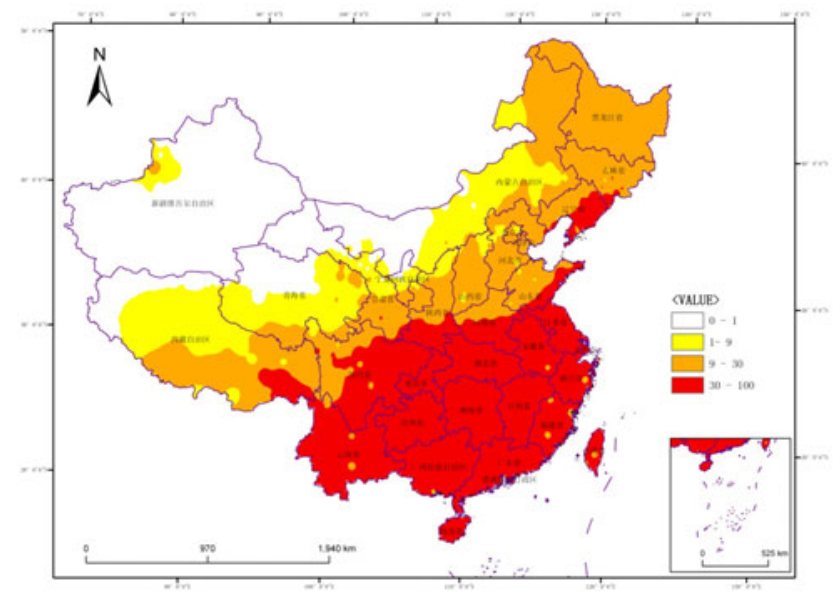

Fig. 1. Potential distribution of Aedes triseriatus in China

\subsection{Potential Distribution of LaCrosse Virus in China}

MaxEnt uses suitable values with the scope of 0-100 as the indices to judge the probability of colonization, and according to the distribution of LAC virus in North America, the suitable values are divided into four risk levels: high, moderate, low and no risk. The result shows that there are widespread suitable distribution areas for this LAC virus in east China. Its highly suitable distribution areas include Heilongjiang, Jilin, Liaoning, Hebei, Beijing, Tianjin, Shandong, Henan, Jiangsu, Anhui, Zhejiang, Hubei, Jiangxi, Fujian, Guangdong, Hunan, south Shanxi, central and south Shaanxi, east Sichuan south Tibet. Its moderate suitable distribution areas include north Heilongjiang, Guangxi and east Guizhou. Its low suitable distribution areas mainly concentrate in Guizhou, Yunnan, south Gansu, north Shaanxi, north Shanxi, north Hebei, east Inner Mongolia and north Xinjiang.

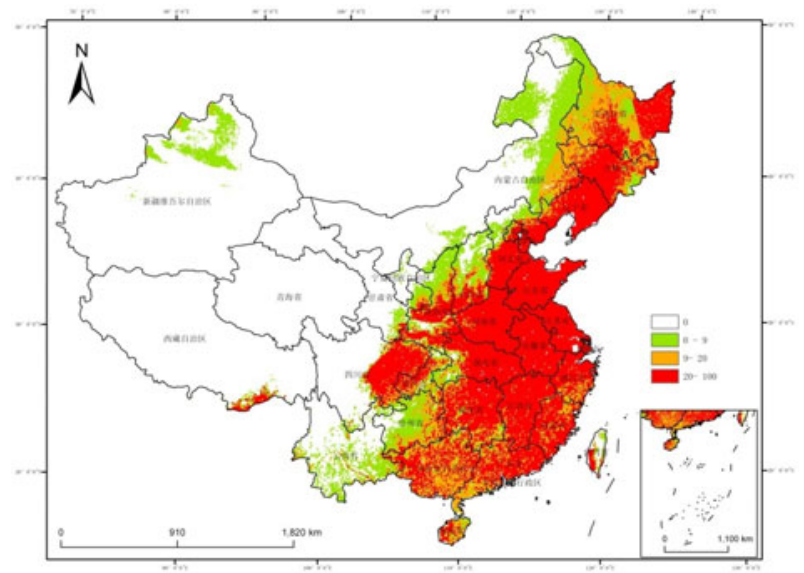

Fig. 2. Potential distribution of LaCrosse encephalitis in China 


\subsection{Result of Risk Analysis for Spread of Aedes triseriatus to China}

As the solely transmission of LAC virus and impossibility spreading without A. triseriatus, the vector insect and the host jointly contribute to the epidemic of LAC virus and decide the risk. Furthermore, during the risk superposition process, the vector's weight result is greater than the virus's weight result. The superposition matrix is shown in the following figure.

Table 2. Risk Matrix

\begin{tabular}{ccccc}
\hline Virus/Vector & High 4 & Moderate 3 & Low 2 & No risk1 \\
\hline High 4 & High 44 & Moderate 34 & Low 24 & No risk 1 \\
Moderate 3 & High 43 & Moderate 33 & Low 23 & No risk 1 \\
Low 2 & High 42 & Moderate 32 & Low 22 & No risk 1 \\
No risk 1 & High 41 & Moderate 31 & Low 21 & No risk 1 \\
\hline
\end{tabular}

According to the matrix analysis, the risks are divided into 4 grades and 13 levels by the vector weight superposition theory. The ArcGIS spatial analysis module is used to realize and demonstrate the above superimposition theory and gain the risk of spread of Aedes triseriatus into China (as shown in Fig. 3).

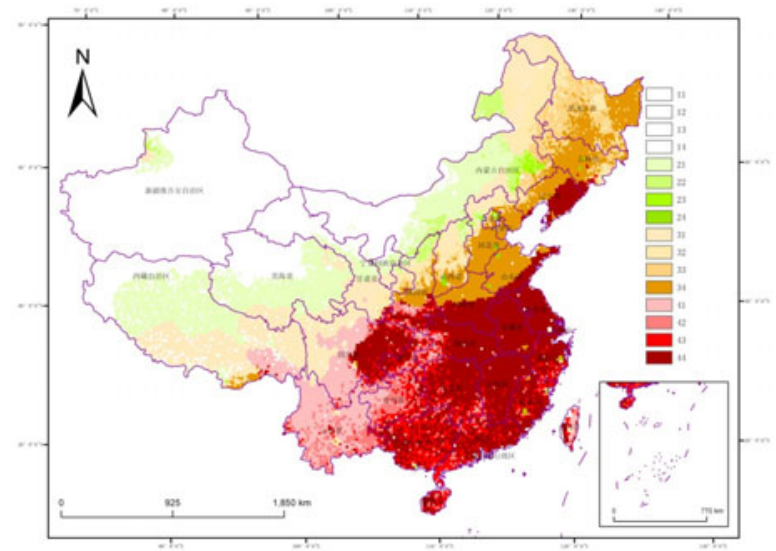

Fig. 3. Potential risk of Aedes triseriatus in China

The result is displayed in four color series: red, yellow, green and colorless, which presents the four risk levels high, moderate, low and no risk of $A$. triseriatus invasion respectively. Each color series is divided into four sublevels from dark to light, reflecting a high-to-low risk tendency in each level. The red areas with risk level of 44, 43, 42 and 41 represent high-risk areas. The risk level is 44 in deep red areas are highest risk level in the high-risk areas, such as east Liaoning, south Shandong, south Henan, Jiangsu, Anhui, Hubei, Hunan, Zhejiang, Jiangxi, Fujian, Guangdong, Guangxi, Chongqing, east Sichuan, Hainan and west Taiwan. The areas with the risk 
levels of 43, 42 and 41 mainly concentrate in Yunnan, central Sichuan, Guizhou and southeast Tibet. The yellow areas 34, 33, 32 and 31 represent moderate-risk areas, and the risk level is 34 in the areas with a comparatively high risk, such as south Shaanxi, south Shanxi, north Hunan, north Shandong, southeast Hebei, Beijing, Tianjin, northwest Liaoning, Jilin, Heilongjiang and southeast Tibet. The areas with risk level of 33, 32 and 31 concentrate in northeast Inner Mongolia, north Hebei, north Shanxi, north Shaanxi, south Gansu, northwest Sichuan, south Tibet and northwest Xinjiang. The green areas with risk level of $24,23,22$ and 21 represent low-risk areas, and the risk level is 24 in areas with a comparatively high risk level, such as east Inner Mongolia, Beijing, Shanxi, Hebei and Shandong. The areas with risk level of 23, 22 and 21 mainly concentrate in north Tibet, south Qinghai, central Gansu, north Ningxia, central Inner Mongolia, north Shanxi and northeast Xinjiang. In the impossible surviving area of vector $A$. triseriatus, the risk superimposition result is $14,13,12$ and 11 which can be regarded as at no risk because the vector cannot survive and the pathogen cannot be transmitted.

\section{Discussion}

In 2005, Craig R Williams et al. used the CLIMEX model which is based on the biological climate theory to predict the potential distribution of culex tritaeniorhynchus which transmitted Japanese encephalitis virus (JEV) in Australia and New Zealand. The predicted distribution result of the mosquito is highly consistent with its actual distribution. This shows that the CLIMEX model can play a good role in predicting the potential distribution of organism vectors. However, the study did not carry out further analysis of the JEV carried by culex tritaeniorhynchus. Kurt D. Reed et al. had predicted the latent distribution areas of Blastomyces dermatitidis using the MaxEnt model.

The study assessed the risk of LAC virus mainly transmitted by Aedes triseriatus using the MaxEnt model. The above two kinds of risk simulation results underwent superposition analysis based on the geographic information system and formed a new model for biological risk analysis of vectors.

Once $A$. triseriatus spreads to China, it will not only annoy people but also pose a threat to their health. In addition, this mosquito can be carried easily, and with increase in trade between China and North America, A. triseriatus is likely to be carried by trading tools such as ship, container. Therefore, it is necessary to formulate the relevant risk management measures according to the risk analysis result to protect people's health.

\section{References}

[1] Williams, D.D., Mackay, S.E., Verdonschot, R.C.M., et al.: Natural and manipulated populations of the treehole mosquito, Ochlerotatus triseriatus, at its northernmost range limit in southern Ontario, Canada. Journal of Vector Ecology 32(2), 328-335 (2007)

[2] Penrod, J.R.: Aedes triseriatus and treeholes: trophic interactions and factors influencing larval growth. Michigan states University, Michigan (2000) 
[3] Clay, M.E., Venard, C.E.: Diapuase in Aedes triseriatus (Diptera: Culicidae) Larvae Terminated by Molting Hormones. Annals of the Entomological Society of America 64(4), 968-970 (1971)

[4] Baker, F.C.: The effect of photoperiodism on resting, treehole, mosquito larvae. Can. Entomol. 67, 149-153 (1935)

[5] Donald, A.S., George, B.C.: Egg diapause in Aedes Triseriatus (Diptera:Culicidae): geographic variation in photoperiodic response and factors influencing diapause termination. J. Med. Entomol. 20(6), 601-607 (1983)

[6] Jalil, M.: Effect of temperature on larval growth of Aedes triseriatus. Journal of Economic Entomology 65(2), 625-626 (1972)

[7] Song, H.M., Zhang, Q.F., Han, X.M.: CLIMEX: Professional biological software for predicting potential distribution of species. Entomological Knowledge 41(4), 379-386 (2004)

[8] Sutherst, R.W., Maywald, G.F.: A computerised system for matching climates in ecology. Agriculture, Ecosystems \& Environment 13(3-4), 281-299 (1985)

[9] Sutherst, R.W.: Implications of global change and climate variability for vector-borne diseases: generic approaches to impact assessments. International Journal for Parasitology 28, 935-945 (1998)

[10] Sutherst, R.W.: Prediction of species geographical ranges. Journal of Biogeography 30, 805-816 (2003)

[11] Sutherst, R.W., Maywald, G.F., Russell, B.L.: Estimating vulnerability under global change modular-modelling of pests. Agriculture, Ecosystems \& Environment 82, 303 319 (2000)

[12] Sutherst, R.W., Maywald, G.F., Bottomley, W., et al.: CLIMEX version2 User Guide. Hearne Scientific Software

[13] Barker, C.M., Paulson, S.L., Cantrell, S., et al.: Habitat preferences and phenology of Ochlerotatus triseriatus and Aedes albopictus (Diptera: Culicidae) in southwestern Virginia. Journal of Medical Entomology 40(4), 403-410 (2003)

[14] Yu, P., Wei, R., Wang, Z.L., Yang, Y.J.: Review and monitoring measures of west nile virus vectors. Chinese Journal of Vector Biology and Control 16(4), 324-326 (2005)

[15] Wu, L., Xie, W.: West Nile Encephalitis. Chinese Journal for Clinicians 34(3), 15-17 (2006) 